\title{
Evaluation of interleukin-1 beta and the ratio of interleukin-1 beta to interleukin-1 receptor antagonist in gingival crevicular fluid during orthodontic canine retraction
}

\author{
Sylwia Moty ${ }^{1, A-D, F,}$, Daniele Manfredini2, ${ }^{2}$, Zuzanna Oruba ${ }^{3, D, F}$, Jolanta Bugajska ${ }^{4, B, C}$, Krystyna Sztefko ${ }^{4, B, C}$, Wojciech Stós ${ }^{1, B}$, \\ Magdalena Osiewicz, ${ }^{5, \mathrm{E}, \mathrm{F}}$, Bartłomiej Wincenty Loster ${ }^{1, \mathrm{E}, \mathrm{F}}$, Frank Lobbezo0 ${ }^{6, \mathrm{E}}$ \\ ${ }^{1}$ Department of Orthodontics, Faculty of Medicine, Jagiellonian University Medical College, Kraków, Poland \\ ${ }^{2}$ School of Dentistry, University of Siena, Italy \\ ${ }^{3}$ Department of Periodonology and Clinical Oral Pathology, Faculty of Medicine, Jagiellonian University Medical College, Kraków, Poland \\ ${ }^{4}$ Institute of Pediatrics, Department of Clinical Biochemistry, Faculty of Medicine, Jagiellonian University Medical College, Kraków, Poland \\ ${ }^{5}$ Department of Integrated Dentistry, Faculty of Medicine, Jagiellonian University Medical College, Kraków, Poland \\ ${ }^{6}$ Department of Oral Kinesiology, Academic Centre for Dentistry Amsterdam (ACTA), University of Amsterdam and Vrije Universiteit Amsterdam, the Netherlands \\ A - research concept and design; $\mathrm{B}$ - collection and/or assembly of data; $\mathrm{C}$ - data analysis and interpretation; \\ $D$ - writing the article; $E$ - critical revision of the article; $F$ - final approval of the article
}

Address for correspondence

Sylwia Motyl

E-mail: sylwia.moty@@uj.edu.pl

Funding sources

None declared

Conflict of interest

None declared

Received on December 15, 2020

Reviewed on January 4, 2021

Accepted on January 31,2021

Published online on March 23, 2021

Cite as

Motyl S, Manfredini D, Oruba Z, et al. Evaluation of interleukin-1 beta and the ratio of interleukin- 1 beta to interleukin-1 receptor antagonist in gingival crevicular fluid during orthodontic canine retraction. Dent Med Probl. 2021;58(1):47-54. doi:10.17219/dmp/132954

DOI

10.17219/dmp/132954

Copyright

○) 2021 by Wroclaw Medical University

This is an article distributed under the terms of the

Creative Commons Attribution 3.0 Unported License (CC BY 3.0)

(https://creativecommons.org/licenses/by/3.0/).

\begin{abstract}
Background. Orthodontic tooth movement (OTM) is a complex phenomenon mediated by cytokines, of which interleukin-1 beta (IL-1B) is potently involved in the remodeling of the periodontal ligament (PDL) and bone. Whether the pattern of IL-1 $1 \beta$ release differs at the sides of tension and compression is not yet clarified.
\end{abstract}

Objectives. The aim of the present study was to evaluate the level of $I L-1 \beta$ and the ratio of $I L-1 \beta$ to interleukin-1 receptor antagonist (IL-1RA) in gingival crevicular fluid (GCF) at the tension and compression sides during orthodontic canine retraction.

Material and methods. Seventeen patients scheduled for orthodontic treatment with bilateral extraction of maxillary first premolars and canine retraction were enrolled. Tooth 2.3 was retracted, teeth 1.3 and 3.3 served as controls. Gingival crevicular fluid samples were collected from the tension and compression sides of each tooth at baseline (before the $7^{\text {st }}$ activation - day 0 ) and at days 2 and 7 , and then again before the $2^{\text {nd }}$ activation (day 28 ) and at days 30 and 35 . The levels of IL-1 $\beta$ and IL-RA were evaluated with the enzyme-linked immunosorbent assay (ELISA).

Results. After the $1^{\text {st }}$ activation, a statistically significant increase in the level of $\mathrm{LL}-1 \beta$ was observed at teeth $2.3(p<0.03$ mesially and $p<0.05$ distally) and 1.3 ( $p<0.05$ mesially and distally), both at the tension and compression sides. The $2^{\text {nd }}$ activation resulted in a gradual increase in the IL-1 13 level at both canines; however, statistical significance was reached only for tooth $2.3(p<0.05$ mesially and $p<0.02$ distally). In terms of the IL-1B/L-1RA ratio, a significant increase was observed only at the compression side of the experimental tooth $(p<0.01)$.

Conclusions. An increase in the IL-1 $1 \beta$ level in GCF was observed both at the tension and compression sides of the actively retracted canine 2.3 as well as the contralateral canine 1.3; a significant rise in the IL-1B/IL-1RA ratio was noted only at the compression side of the experimental tooth 2.3, indicating the zone of active bone resorption.

Key words: interleukin-1 beta, orthodontic tooth movement, canine retraction 


\section{Introduction}

Orthodontic tooth movement (OTM) is based on the periodontal ligament (PDL) and alveolar bone remodeling induced by an external force exerted by an orthodontic appliance. ${ }^{1}$ Sterile inflammation is the essence of this biological response. ${ }^{2}$ Migrating immune cells and native cells residing in PDL synthesize and release a plethora of cytokines, growth factors, and metabolites of arachidonic acid., Although not fully elucidated, their hypothesized role is to mediate tooth movement by promoting the differentiation, maturation and activation of cells responsible for bone remodeling. ${ }^{5,6}$

Many studies have shown elevated levels of several inflammatory cytokines, such as interleukin-1 beta (IL-1 $\beta$ ), interleukin-6 (IL-6), interleukin-8 (IL-8), and tumor necrosis factor alpha (TNF- $\alpha$ ), in gingival crevicular fluid (GCF) upon the application of the orthodontic force to the tooth. ${ }^{7-9}$ Among those, Il-1 $\beta$, a key mediator in a variety of activities in immune and acute-phase inflammatory responses, ${ }^{10}$ seems to be one of the most potent mediators in the process of periodontal tissue remodeling at the initial stages of OTM. ${ }^{11,12}$ Its major role is to activate osteoclasts and promote their fusion and survival. ${ }^{11,12}$ It has been shown that upon the application of the retracting force to the maxillary canine, the level of IL-1 $\beta$ in GCF at the distal aspect of the tooth elevates, reaching its peak upon $24-72 \mathrm{~h} \cdot{ }^{13,14}$ Also, a positive correlation between the Il-1 $\beta$ level in GCF and the rate of OTM has been described. ${ }^{15,16}$ In vivo, the biological activity of IL-1 $\beta$ is controlled by a naturally occurring antagonist of its receptor, interleukin-1 receptor antagonist (IL-1RA). The application of mechanical stress to the tooth is related not only to an absolute increase in IL-1 $\beta$ secretion, but also to an elevated value of the IL-1 $\beta /$ IL-1RA ratio. ${ }^{15}$ Interleukin-l beta also stimulates the production of IL- 6 , which, just like IL-1 $\beta$, expresses synergistic activity. ${ }^{11}$

It is well documented that pro-inflammatory cytokines are involved in bone resorption and the inhibition of bone formation. ${ }^{15}$ Thus, they facilitate OTM by acting particularly at the pressure side. ${ }^{11}$ Probably, a distinct subset of cytokines plays a role in bone apposition at the side of tension. Within these premises, the majority of studies on canine retraction evaluated the cytokine content of GCF only at the side of compression. ${ }^{13,17}$ The pattern of their release and their potential role in bone apposition at the tension side are not yet clarified. Therefore, the aim of the present study was to evaluate the GCF level of IL- $1 \beta$ and the ratio of IL- $1 \beta$ to IL-1RA at the compression and tension sides during OTM (canine retraction) in adolescents. The null hypothesis of the study was that there is no difference between the tension and compression sides in terms of either the concentration of IL- $1 \beta$ or the IL-1 $\beta /$ IL-1RA ratio in GCF.

\section{Material and methods}

\section{Study population}

Seventeen patients ( 8 males and 9 females) aged from 13 to 18 years (mean age: $16 \pm 4$ years) scheduled to start orthodontic treatment at the Department of Orthodontics, Dental University Clinic in Kraków, Poland, were enrolled to the study. The participants had to meet the following inclusion criteria: 1 . no systemic diseases; 2 . no use of systemic antibiotic therapy within 2 weeks prior to inclusion; 3. no use of systemic anti-inflammatory drugs within 1 week prior to inclusion; 4. good periodontal health (i.e., the bleeding on probing index (BOP) value not exceeding 10\%, no probing depths exceeding $2 \mathrm{~mm}$ and no bone loss in the radiographic examination); and 5. the necessity for canine retraction in the course of orthodontic treatment. Ineligibility with respect to any of the inclusion criteria and/or no consent to the enrollment to the study, expressed by either the patient and/or the parents, constituted the basis for exclusion. Among the patients of the study group, the following malocclusions were present: maxillary and mandibular crowding (9 patients); disto-occlusion (1 patient); disto-occlusion with maxillary and mandibular crowding (5 patients); an open bite ( 1 patient); and the agenesis of maxillary lateral incisors (1 patient).

The study protocol was reviewed and approved by the Bioethical Committee (approval No. KBET/466/B/2003). All patients and their parents gave written informed consent to participation upon receiving detailed explanation of the aim and course of the study.

\section{Course of orthodontic treatment}

Careful anamnesis, physical examination, analysis of cast models, orthopantomograms (OPGs), and cephalometric radiographs were carried out for each patient. The treatment plan for the included patients, except for the one with the agenesis of lateral incisors, involved the extraction of maxillary first premolars. All patients were scheduled for maxillary canine retraction. Upon the extractions of premolars, orthodontic separators were placed in the maxillary arch. One week later, bands were bonded on the maxillary first molars. Metal brackets (Victory Series ${ }^{\mathrm{TM}}$ MBT .022"; 3M Unitek, Maplewood, USA) were bonded onto the teeth in the upper arch. Additionally, all patients received transpalatal arches (TPAs) bended from a 0.9-millimeter wire in order to prevent the mesial movement of first molars during active canine retraction. The retraction of canines was performed according to an individual orthodontic treatment plan based on a careful examination of each patient, and the analysis of OPGs, cephalometric radiographs and plaster models. 
In each patient, the retraction was firstly initiated for the upper left canine (tooth 2.3), which was the experimental tooth. The retraction of the contralateral canine (tooth 1.3) was delayed by 2 months, until the end of the experimental procedure (GCF collection - see below). Teeth 1.3 and 3.3 served as controls.

All patients received also orthodontic treatment in the lower arch by means of fixed orthodontic appliances upon the completion of the experimental procedure in order to ensure proper occlusal relations. Orthodontic treatment lasted around 2 years in each patient. Upon the completion of the treatment, all patients were provided with a removable splint for the maxillary arch and a fixed orthodontic retainer in the mandibular arch.

\section{Experimental procedure - recorded parameters}

In each patient, GCF was collected from the mesial and distal aspects of the experimental tooth 2.3 and the control teeth (1.3 and 3.3). The GCF collection was performed initially before the $1^{\text {st }}$ activation of the appliance (day 0), and $48 \mathrm{~h}$ (day 2) and $168 \mathrm{~h}$ (day 7) after the $1^{\text {st }}$ activation. After 4 weeks, GCF was sampled again before the $2^{\text {nd }}$ activation (day 28), and $48 \mathrm{~h}$ (day 30) and $168 \mathrm{~h}$ (day 35 ) after the $2^{\text {nd }}$ activation. The study protocol is presented on the flowchart (Fig. 1).

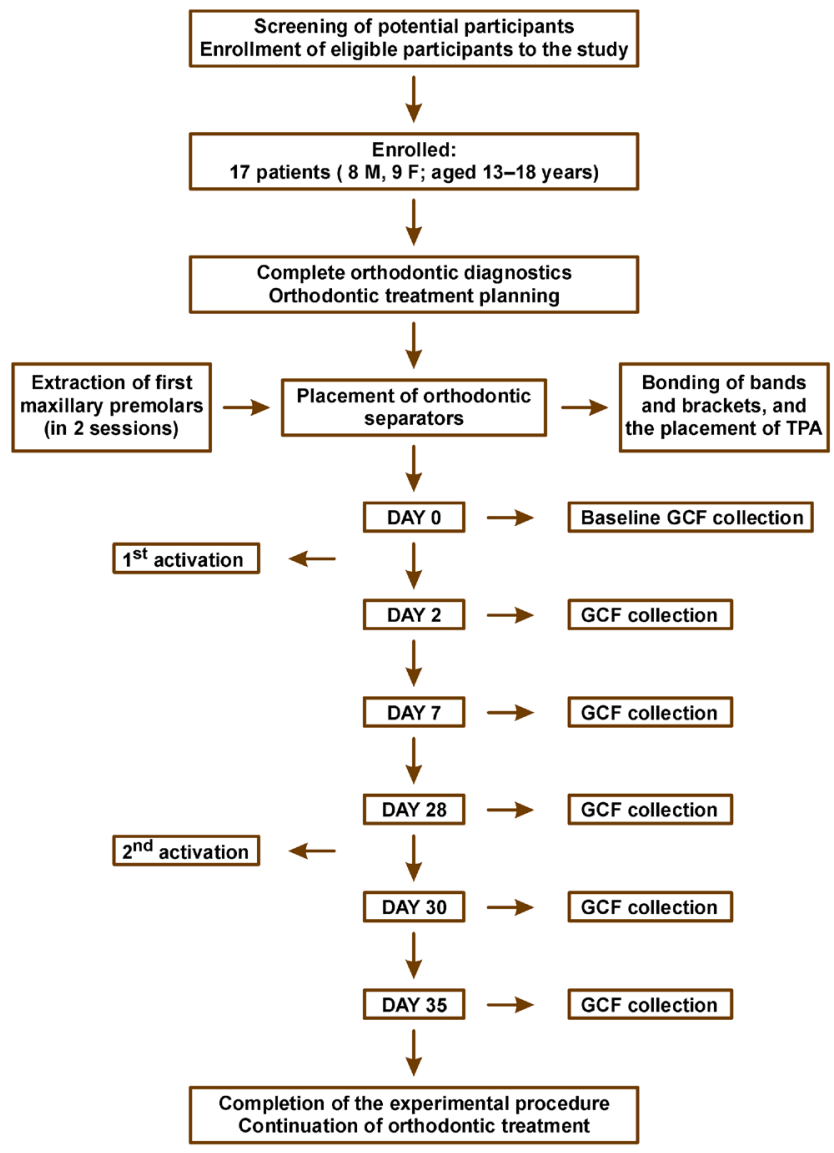

Fig. 1. Flowchart of the experimental protocol

M - males; F - females; TPA - transpalatal arch; GCF - gingival crevicular fluid.

\section{GCF collection}

Each patient received detailed oral hygiene instructions prior to the placement of brackets, and was motivated to reinforce oral hygiene throughout the entire experimental period in order to minimize the influence of gingival inflammation on the cytokine levels. Additionally, the patients were instructed to rinse their mouth with $0.1 \%$ chlorhexidine mouthwash for 1 min twice a day, for 2 weeks prior to the GCF collection procedure.

At the appointments for the GCF collection, the sampling teeth were isolated with cotton rolls and suction, cleaned with cotton pellets soaked in distilled water and gently dried with air from an air syringe. Gingival crevicular fluid was collected on sterile absorbent filter-paper strips (PerioPaper ${ }^{\circledR}$ GCF Collection Strips; Oraflow, Plainview, USA). In brief, the PerioPaper strip was grasped by holding the orange handle with pliers and its white portion was gently inserted to the gingival crevice until a minimum of resistance was felt, and it was kept in situ for $30 \mathrm{~s}$. During each appointment, a maximum of 3 strips were inserted at each side at 1-minute intervals in order to collect a specified volume of fluid, sufficient for the biochemical analysis. In total, a maximum of 3 separate strips were collected from the mesial, and 3 from the distal aspect of the experimental tooth 2.3. Similar procedures were performed at the control teeth $(1.3,3.3)$ in each patient at each time-point of the study. Directly upon collection, the volume of GCF on each strip was determined using Periotron $8000^{\circledR}$ (Oraflow). Prior to measurements, the instrument was carefully calibrated, and a standard calibration curve was constructed using a range of volumes of human serum of known biochemical compounds' concentrations. Following volume determination, the strips were placed in sterile cryo-tubes and kept in $-70^{\circ} \mathrm{C}$ until the biochemical analysis.

\section{Biochemical analysis}

When the process of GCF collection was completed in all patients, the samples were defrosted, and the strips collected from the same aspect (either mesial or distal) of the same tooth at the same time-point were pooled together in a vial. The fluid was eluted from the strips as follows: $110 \mu \mathrm{L}$ of Tris- $\mathrm{HCl}$ buffer ( $\mathrm{pH} 7.5$ ) was added to each vial and the vials were shaken on an orbital shaker for $10 \mathrm{~min}$; subsequently, the vials were centrifuged at 1,500 $\mathrm{g}$ for $5 \mathrm{~min}$. The supernatant was placed in a fresh vial. This procedure was repeated to gather the proper volume, and the end volume of the eluate was $200 \mu \mathrm{L}$.

The concentration of IL-1 $\beta$ and IL-1RA in the eluates was determined by means of the immunochemical method - the enzyme-linked immunosorbent assay (ELISA) (BioSource, Ottignies-Louvain-la-Neuve, Belgium; R\&D Systems, Minneapolis, USA, respectively). Additionally, the total protein concentration was determined spectrophotometrically. 


\section{Data analysis}

Patient enrollment, and all the procedures related to orthodontic treatment and the GCF collection were performed by one examiner (S.M.).

For each GCF sample, the degree of eluation with Tris- $\mathrm{HCl}$ buffer was calculated based on the initial sample volume (measured by means of Periotron 8000) and the known volume of the added buffer. This dilution was taken into account when calculating the concentrations of cytokines and the total protein concentration. The concentrations of cytokines were calculated in relation to the protein concentration $[\mathrm{pg} / \mu \mathrm{g}]$. The obtained results were presented as mean $(M)$ \pm standard deviation $(S D)$ for the mesial and distal sides of each examined tooth. Changes in the cytokine levels for each tooth at different time-points were compared by means of Student's $t$ test upon the confirmation of normal distribution. The level of statistical significance was set at $p<0.05$.

\section{Results}

All 17 patients completed the study. None of the study participants exhibited gingival inflammation during the experimental period.

\section{GCF total protein concentration}

Figure 2 presents the total protein concentration in GCF at the experimental (2.3) and control (1.3 and 3.3) teeth. An increase in the total protein concentration in GCF was observed at all evaluated teeth upon the bonding of braces, i.e., the $1^{\text {st }}$ application of the orthodontic force and the activation of the experimental canine. This increase reached statistical significance with respect to baseline at days $2(p<0.020)$ and $7(p<0.001)$ for the mesial aspect of the control tooth 1.3, and at day 7 for the mesial aspect of tooth $2.3(p<0.005)$. Of note, a rise in total protein in GCF was also significant for the control tooth 3.3, reflecting the general response within the oral cavity to the occurrence of the orthodontic force. Following the $2^{\text {nd }}$ activation, the level of protein dropped, and this effect was also noticeable for all evaluated teeth.

\section{GCF concentration of IL-1 $\beta$}

The GCF concentration of IL-1 $\beta$ at the experimental (2.3) and control (1.3 and 3.3) teeth is presented in Fig. 3. Upon the $1^{\text {st }}$ activation of the retracted canine 2.3 , a statistically significant increase in the level of IL-1 $\beta$ in relation to total protein was observed at day 2 , both for the mesial and distal aspect of this tooth $(p<0.030$ and $p<0.050$, respectively). At day 7 , a drop in IL- $1 \beta /$ total protein was observed at both sides; however, it reached statistical significance only at the mesial aspect $(p<0.010)$. Following the $2^{\text {nd }}$ activation, a significant increase in IL-1 $\beta /$ total protein was observed at day 7 (experimental day 35), mesially and distally $(p<0.050$ and $p<0.020$, respectively).

Similar changes in the amount of IL- $1 \beta$ in relation to the total protein content in GCF were observed at the control
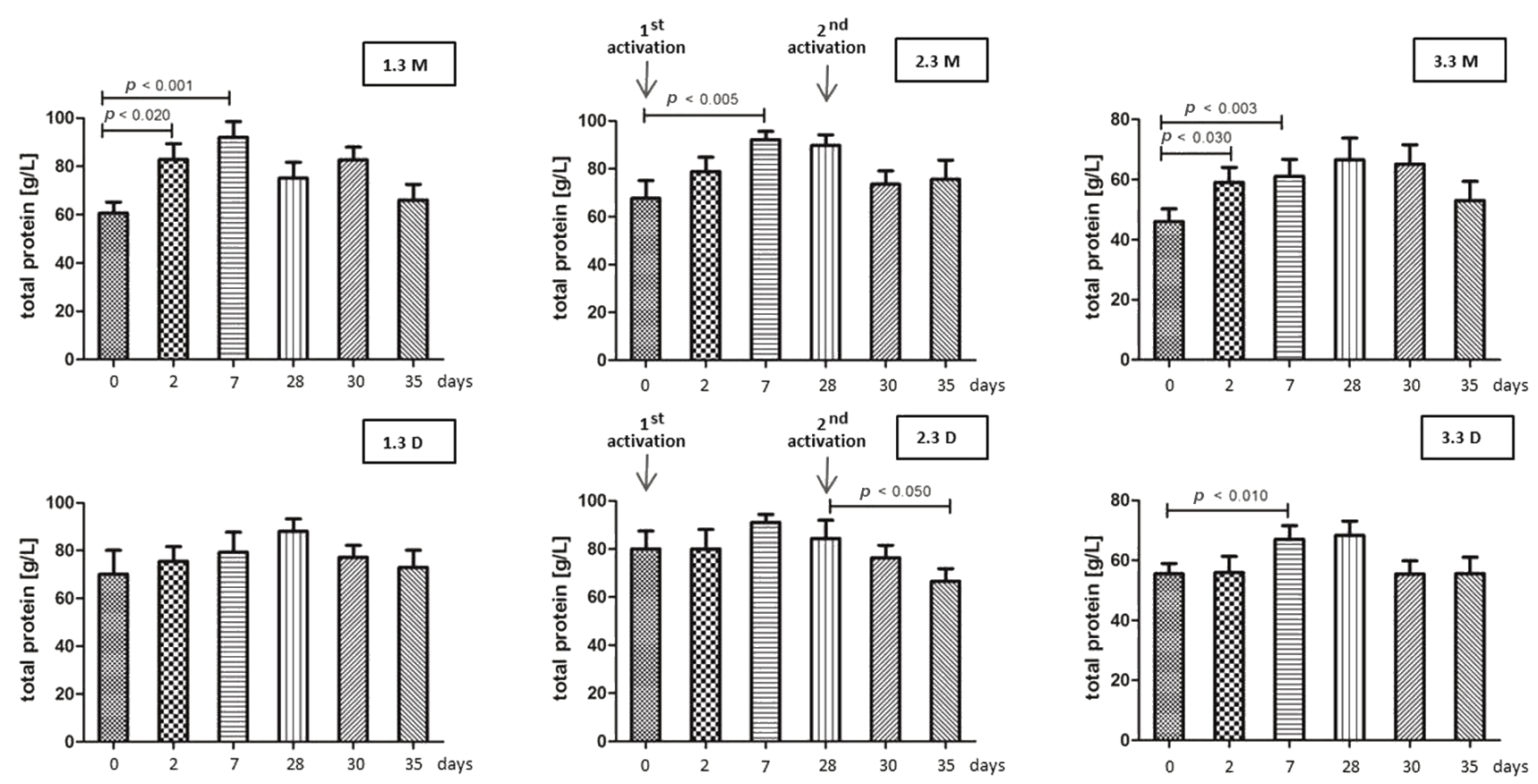

Fig. 2. Mean total protein concentration at the evaluated teeth $[\mathrm{g} / \mathrm{L}]$

Error bars indicate standard deviation (SD); symbols in the upper right frames at each chart represent tooth number (1.3, 2.3, 3.3) and the evaluated side (M - mesial, D - distal) 

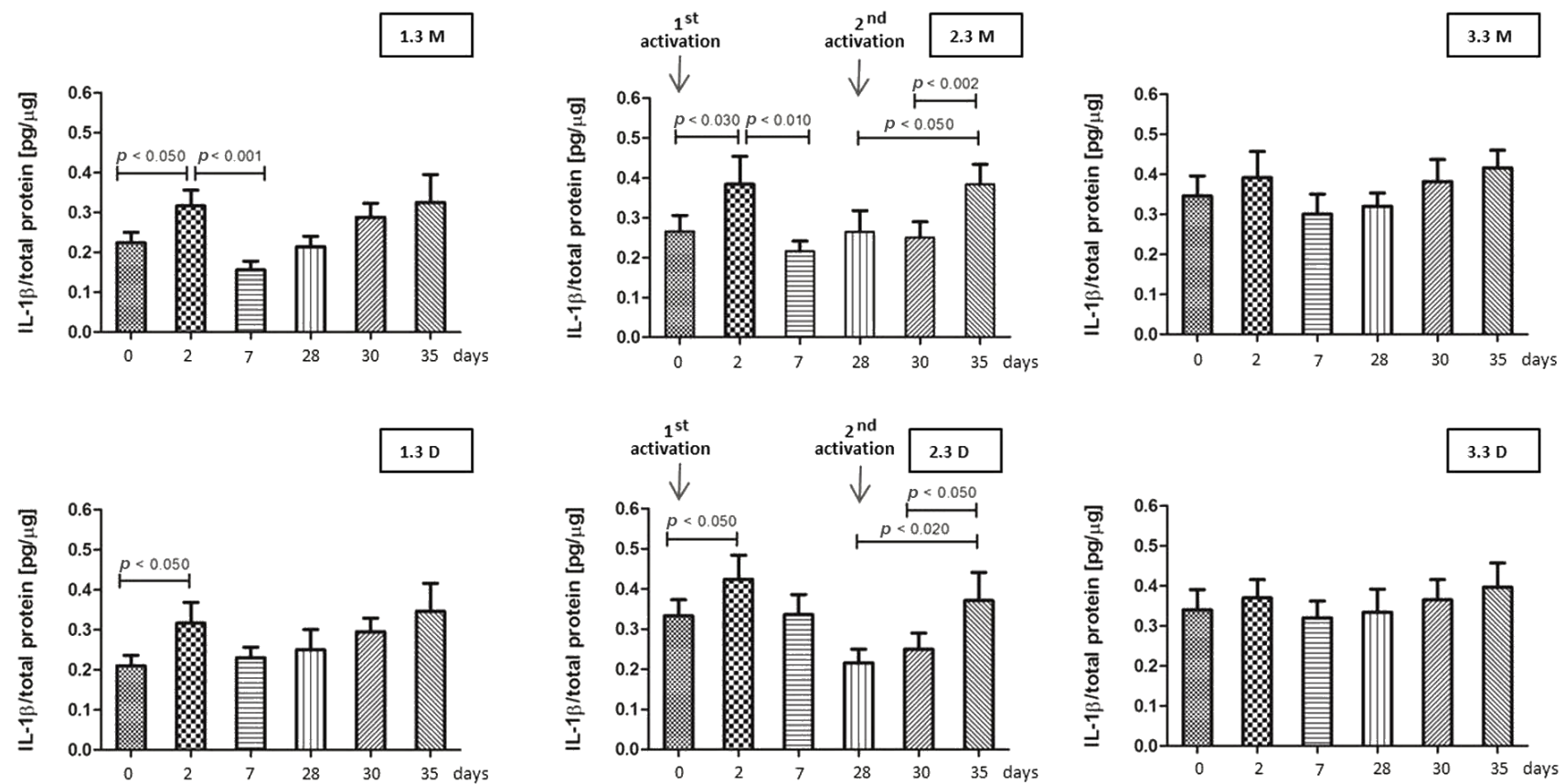

Fig. 3. Mean concentration of interleukin-1 beta $(\mathrm{IL}-1 \beta)$ in relation to total protein $[\mathrm{pg} / \mu \mathrm{g}]$

Error bars indicate standard deviation (SD); symbols in the upper right frames at each chart represent tooth number (1.3, 2.3, 3.3) and the evaluated side ( $M$ - mesial, D - distal).

tooth 1.3, i.e., a rapid and significant increase on day 2 after the $1^{\text {st }}$ application of the orthodontic force $(p<0.050$ for both the mesial and distal aspect) with a subsequent, albeit not significant (except for the mesial aspect, with $p<0.001)$, decrease at day 7 , and a tendency to a gradual increase throughout the 7 -day period upon the $2^{\text {nd }}$ activation.
No significant changes in IL- $1 \beta /$ total protein were observed for the control tooth 3.3.

\section{GCF IL-1 $\beta$ to IL-1RA ratio}

The ratio of IL-1 $\beta$ to IL-1RA in GCF at the experimental (2.3) and control (1.3 and 3.3) teeth is presented in Fig. 4.
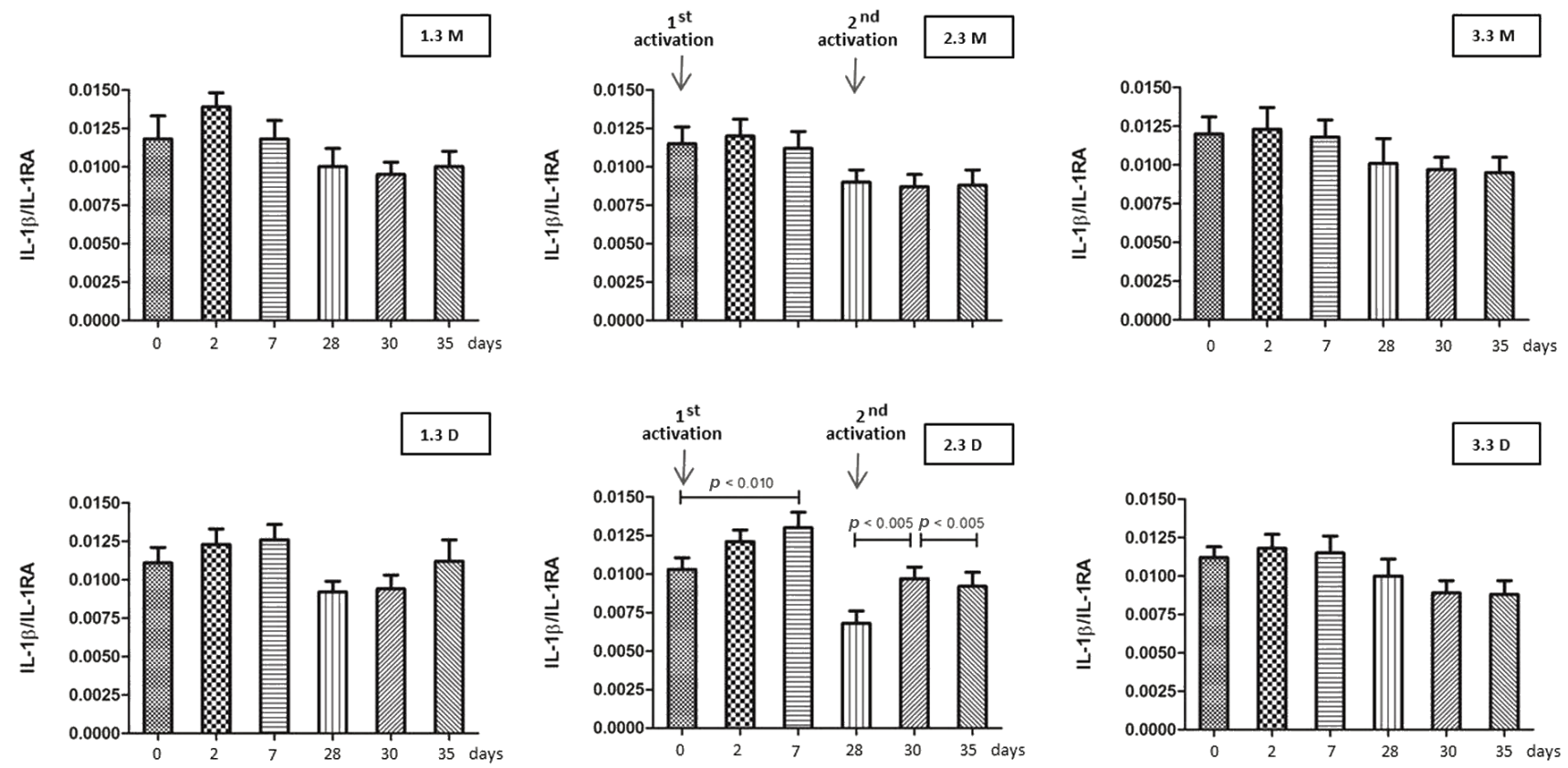

Fig. 4. Mean IL-1 $\beta$ to interleukin-1 receptor antagonist (IL-1RA) ratio

Error bars indicate standard deviation (SD); symbols in the upper right frames at each chart represent tooth number (1.3, 2.3, 3.3) and the evaluated side (M - mesial, D - distal). 
Interestingly, statistically significant changes in this ratio were observed only for the distal aspect, i.e., the compression side, of the experimental tooth. Upon the $1^{\text {st }}$ activation, a gradual increase in IL-1 $\beta /$ IL-1RA was noted throughout the following 7 days, and it reached statistical significance at day $7(p<0.01)$. Following the $2^{\text {nd }}$ activation, a rapid rise in IL-1 $\beta /$ IL-1RA took place at day 2 (experimental day 30) $(p<0.005)$, and the level of this relation remained relatively stable until day 7 (experimental day 35). In turn, no significant changes in IL-1 $\beta / I L-1 R A$ were observed at the mesial aspect, i.e., the tension side, of the experimental tooth 2.3 as well as at the control teeth (1.3 and 3.3), both mesially and distally.

\section{Discussion}

The application of the orthodontic force to a tooth results in a series of orchestrated cellular and molecular events, leading to the remodeling of periodontal tissues. ${ }^{18}$ Within this remodeling, 2 distinct processes take place simultaneously at the opposite sides of the moved tooth, i.e., bone resorption at the pressure side and bone deposition at the tension side. A bunch of cytokines, of which pro-inflammatory IL- $1 \beta$ is the most potently associated with resorption, mediate these phenomena. Indeed, IL-1 $\beta$ induces the expression of the receptor activator of the nuclear factor in osteoblasts and PDL cells, and stimulates the differentiation of osteoclast precursors as well as the fusion, survival and activation of matured osteoclasts. ${ }^{2,16,19}$ Hence, its role seems of particular importance at the compressed areas of PDL, rather than at the sides where tension occurs. Thus, IL- $1 \beta$ has been proposed as a biological marker of tissue remodeling. The biological effects of IL-1 $\beta$ are, however, controlled by a naturally occurring antagonist of its receptor, IL-1RA. ${ }^{20}$ Thus, monitoring the IL- $1 \beta / \mathrm{IL}-1 \mathrm{RA}$ ratio gives the most credible view on the intensification of resorptive processes. Based on this premise, the aim of the present study was to evaluate the level of IL- $1 \beta$ and the IL-1 $\beta /$ IL-1RA ratio in GCF at the initial stages of OTM (canine retraction), at the sides of compression and tension.

An increase in the total protein content in GCF was observed within the $1^{\text {st }}$ week upon the $1^{\text {st }}$ application of the orthodontic force at the experimental tooth as well as at the control teeth. This is an interesting observation and suggests that the introduction of an orthodontic appliance to the oral cavity, although initially bonded only in one dental arch, induces a generalized cascade of events in terms of upregulation of gene expression and protein synthesis in periodontal tissues.

At the sides around the retracted canine 2.3, an increase in the IL- $1 \beta$ concentration was observed both mesially and distally, following the $1^{\text {st }}$ (a rapid rise within the first $48 \mathrm{~h}$, with a drop toward a level similar to baseline at day 7 ) and the $2^{\text {nd }}$ activation (a gradual increase over the following 7 days). Interestingly, a significant increase in the IL-1 $\beta /$ IL-1RA ratio was noted only at the distal aspect of tooth 2.3 , indicating that the compression side of the retracted canine is characterized by the highest potential of bone resorption. Although at the mesial aspect of the retracted canine, the level of IL-1 $\beta$ was elevated after the activations, the IL-1 $\beta /$ IL-1RA ratio exhibited stable values, indicating controlled bone metabolism without a tendency toward resorption.

An increase in the IL-1 $\beta$ concentration was observed also at the control canine 1.3. This can be attributed to the fact that the orthodontic force was distributed within the whole upper dental arch due to the presence of a fixed orthodontic appliance and the retraction of tooth 2.3, eliciting a biologic response at all the teeth incorporated to the appliance. However, no significant alterations of the IL-1 $\beta /$ IL-1RA ratio were noted at this tooth.

No significant changes in either the IL- $1 \beta$ concentration or the $1 \beta / \mathrm{IL}-1 \mathrm{RA}$ ratio were noted at the control tooth 3.3.

A similar pattern of the upregulation of this cytokine at the side of compression in the course of canine retraction has been reported in other studies. ${ }^{13,14,21,22}$ Researchers described an elevation of the IL- $1 \beta$ concentration in GCF in the early phases of canine distalization, with peaks at either $24 \mathrm{~h}^{13,21,22}$ or $72 \mathrm{~h},{ }^{14}$ and with a subsequent decrease toward baseline levels at $168 \mathrm{~h}$. Among the above-mentioned studies, only Uematsu et al. used both the contralateral and antagonist canines as controls, ${ }^{21}$ whereas in the other investigations, only the tooth contralateral to the retracted one served as a control. ${ }^{13,14,22}$ Of note, Uematsu et al. did not observe an elevation of the IL- $1 \beta$ concentration for the contralateral canine, ${ }^{21}$ which was instead shown in our study. In all those studies, the concentration of Il-1 $\beta$ was measured either exclusively at the compression side ${ }^{13}$ or without side specification, ${ }^{14,21,22}$ contrarily to our study, in which the cytokine level was specifically assessed both at the compression and tension sides. In turn, Iwasaki et al. compared the IL-1 $\beta$ level at the mesial and distal sides of the retracted canines and reported a higher increase at the compression side. ${ }^{15}$ The activity of IL-1 $\beta$ remains under the control of IL-1RA. It has been shown that lower values of the IL-1RA concentration and higher values of the IL-1 $\beta /$ IL-1RA ratio are associated with a higher velocity of tooth movement during orthodontic treatment. ${ }^{23}$ An investigation described higher values of IL-1 $\beta / \mathrm{IL}-1 \mathrm{RA}$ at the distal aspect of the retracted canine than at its mesial aspect, ${ }^{9}$ which is in accordance with the outcomes of the present study.

Based on the current knowledge on the effects of the IL-1 $\beta$ upregulation on the velocity of tooth movement, attempts have been made to further increase the synthesis of IL-1 $\beta$ in order to accelerate OTM. Varella et al. showed that the application of low-level laser therapy with a gallium-aluminiumarsenide semiconductor laser during orthodontic treatment contributes to increasing the level of IL-1 $\beta$ in GCF and significantly accelerates tooth translation. ${ }^{16}$ 
The application of the orthodontic force induces the upregulation of a plethora of pro-inflammatory cytokines and enzymes, which act synergistically in the process of tooth movement. Ren et al. pointed that also IL-6, IL- 8 and TNF- $\alpha$ play a significant role at the early stages of tooth movement. ${ }^{9}$ Grant et al. compared cytokine profiles at the compression and tension sides during maxillary canine retraction and noted that while both sides were characterized by increases in the concentration of IL-1 $\beta$, IL-8, TNF- $\alpha$, and matrix metallopeptidase-9 (MMP-9), they differed in terms of tissue inhibitor of matrix metalloproteinase-1 (TIMP-1) and tissue inhibitor of matrix metalloproteinase-2 (TIMP-2) (expressed in higher values at the tension side), and receptor activator of nuclear factor kappa-B ligand (RANKL) (expressed in a higher value on the compression side). ${ }^{24}$ The distal movement of the canine has also been related to the rise in the activity of alkaline phosphatase at the initial stages of treatment. ${ }^{25}$

To the best of the authors' knowledge, this is the only study in which the GCF concentration of IL-1 $\beta$ as well as IL-1 $\beta /$ IL-1RA were evaluated at both sides, i.e., compression and tension, and compared to the contralateral and antagonist canines. Although increased IL-1 $\beta$ synthesis was observed at the compression and tension sides of the retracted and contralateral canines, a significant imbalance between the concentration of this cytokine and its antagonist was observed exclusively at the compressed side of the retracted tooth.

With respect to the clinical relevance of the presented research findings, it should be noted that measuring the levels of IL-1 $\beta$ and IL-1RA could potentially be applied for the determination of proper levels of orthodontic forces in order to obtain desirable velocity of tooth movement. Predicting OTM requires controlled bone resorption, so the rate of the IL-1 $\beta$ and IL-1RA synthesis might be an important indicator of this phenomenon. To this end, future studies may focus on determining the relation between IL-1 $/$ IL-1RA and the rate of OTM.

\section{Limitations}

The results of this study should, however, be carefully considered in the light of several limitations. The main shortcoming is a relatively small study group. The fact that the number of participants was low might have influenced the magnitude of significance for the observed associations. Consequently, the findings of this research should be interpreted cautiously. Secondly, we did not measure the rate of canine retraction upon the application of the orthodontic force, so we were not able to determine whether an increase in the IL- $1 \beta$ level and in the IL-1 $\beta /$ IL-1RA ratio indeed correlated with the velocity of this process. It may also be debatable if differences on the biochemical level between the pressure and tension sides of a single tooth can be precisely shown due to close proximity. Therefore, further clinical studies on larger populations and with well-designed protocols are essential to elucidate the molecular mechanisms responsible for OTM.

\section{Conclusions}

Based on the obtained results, it can be concluded that the protein content in GCF increases in response to the introduction of the orthodontic force and this response takes place in the arch incorporated to the orthodontic appliance as well as in the opposite arch. The application of the orthodontic force to a tooth leads to a rapid increase in the IL- $1 \beta$ level in GCF at the early stages of treatment, at both compression and tension sides, with the subsequent normalization of its concentration. The application of the distalizing force to a tooth is related to a rise in the IL-1 $/$ IL-1RA ratio at the compression side, indicating active bone resorption.

\section{ORCID iDs}

Sylwia Motyl @ https://orcid.org/0000-0003-2378-7726 Daniele Manfredini (1) https://orcid.org/0000-0002-4352-3085 Zuzanna Oruba (1) https://orcid.org/0000-0001-6370-7538 Jolanta Bugajska (1) https://orcid.org/0000-0003-4290-8714 Krystyna Sztefko (1) https://orcid.org/0000-0002-0455-0662 Wojciech Stós (1) https://orcid.org/0000-0003-4836-5562 Magdalena Osiewicz (i) https://orcid.org/0000-0002-8108-4546 Bartłomiej Wincenty Loster (1) https://orcid.org/0000-0001-5724-5808 Frank Lobbezoo (1) https://orcid.org/0000-0001-9877-7640

\section{References}

1. Li Y, Jacox LA, Little SH, Ko CC. Orthodontic tooth movement: The biology and clinical implications. Kaohsiung J Med Sci. 2018:34(4):207-214. doi:10.1016/j.kjms.2018.01.007

2. Teixeira CC, Khoo E, Tran J, et al. Cytokine expression and accelerated tooth movement. J Dent Res. 2010;89(10):1135-1141. doi:10.1177/0022034510373764

3. Krishnan V, Davidovitch Z. Cellular, molecular, and tissue-level reactions to orthodontic force. Am J Orthod Dentofacial Orthop. 2006;129(4):469.e1-e32. doi:10.1016/j.ajodo.2005.10.007

4. Ren Y, Vissink A. Cytokines in crevicular fluid and orthodontic tooth movement. Eur J Oral Sci. 2008;116(2):89-97. doi:10.1111/j.16000722.2007.00511.x

5. Yao Z, Xing L, Qin C, Schwarz EM, Boyce BF. Osteoclast precursor interaction with bone matrix induces osteoclast formation directly by an interleukin-1-mediated autocrine mechanism. J Biol Chem. 2008;283(15):9917-9924. doi:10.1074/jbc.M706415200

6. Yamaguchi M. RANK/RANKL/OPG during orthodontic tooth movement. Orthod Craniofac Res. 2009;12(2):113-199. doi:10.1111/j.16016343.2009.01444.x

7. Alhashimi N, Frithiof $L$, Brudvik $P$, Bakhiet M. Orthodontic tooth movement and de novo synthesis of proinflammatory cytokines. Am J Orthod Dentofacial Orthop. 2001;119(3):307-312. doi:10.1067/ $\bmod .2001 .110809$

8. Garlet TP, Coelho U, Silva JS, Garlet GP. Cytokine expression pattern in compression and tension sides of the periodontal ligament during orthodontic tooth movement in humans. Eur J Oral Sci. 2007;115(5):355-362. doi:10.1111/j.1600-0722.2007.00469.x

9. Ren Y, Hazemeijer H, de Haan B, Qu N, de Vos P. Cytokine profiles in crevicular fluid during orthodontic tooth movement of short and long durations. J Periodontol. 2007;78(3):453-458. doi:10.1902/ jop.2007.060261

10. Dinarello CA. Interleukin-1 and its biologically related cytokines. Adv Immunol. 1989;44:153-205. doi:10.1016/s0065-2776(08)60642-2 
11. d'Apuzzo F, Cappabianca $S$, Ciavarella D, Monsurrò A, SilvestriniBiavati A, Perillo L. Biomarkers of periodontal tissue remodeling during orthodontic tooth movement in mice and men: Overview and clinical relevance. ScientificWorldJournal. 2013;2013:105873. doi:10.1155/2013/105873

12. Davidovitch Z, Nicolay OF, Ngan PW, Shanfeld JL. Neurotransmitters, cytokines, and the control of alveolar bone remodeling in orthodontics. Dent Clin North Am. 1988;32(3):411-435. PMID:2900159

13. Lee KJ, Park YC, Yu HS, Choi SH, Yoo YJ. Effects of continuous and interrupted orthodontic force on interleukin-1beta and prostaglandin E2 production in gingival crevicular fluid. Am J Orthod Dentofacial Orthop. 2004;125(2):168-177. doi:10.1016/j.ajodo.2003.03.006

14. Tian YL, Xie JC, Zhao ZJ, Zhang Y. Changes of interlukin-1beta and tumor necrosis factor-alpha levels in gingival crevicular fluid during orthodontic tooth movement [in Chinese]. Hua Xi Kou Qiang Yi Xue Za Zhi. 2006;24(3):243-245. PMID:16845961

15. Iwasaki LR, Haack JE, Nickel JC, Reinhardt RA, Petro TM. Human interleukin-1 beta and interleukin-1 receptor antagonist secretion and velocity of tooth movement. Arch Oral Biol. 2001;46(2):185-189. doi:10.1016/s0003-9969(00)00088-1

16. Varella AM, Revankar AV, Patil AK. Low-level laser therapy increases interleukin- $1 \beta$ in gingival crevicular fluid and enhances the rate of orthodontic tooth movement. Am J Orthod Dentofacial Orthop. 2018;154(4):535-544.e5. doi:10.1016/j.ajodo.2018.01.012

17. Iwasaki LR, Gibson CS, Crouch LD, Marx DB, Pandey JP, Nickel JC. Speed of tooth movement is related to stress and IL-1 gene polymorphisms. Am J Orthod Dentofacial Orthop. 2006;130(6):698.e1-e9. doi:10.1016/j.ajodo.2006.04.022

18. Kapoor P, Kharbanda OP, Monga N, Miglani R, Kapila S. Effect of orthodontic forces on cytokine and receptor levels in gingival crevicular fluid: A systematic review. Prog Orthod. 2014;15(1):65. doi:10.1186/s40510-014-0065-6

19. Saito M, Saito S, Ngan PW, Shanfeld J, Davidovitch Z. Interleukin 1 beta and prostaglandin $\mathrm{E}$ are involved in the response of periodontal cells to mechanical stress in vivo and in vitro. Am J Orthod Dentofacial Orthop. 1991;99(3):226-240. doi:10.1016/0889-5406(91)70005-H

20. Iwasaki LR, Crouch LD, Tutor A, et al. Tooth movement and cytokines in gingival crevicular fluid and whole blood in growing and adult subjects. Am J Orthod Dentofacial Orthop. 2005;128(4):483-491. doi:10.1016/j.ajodo.2004.03.037

21. Uematsu S, Mogi M, Deguchi T. Interleukin (IL)-1 beta, IL-6, tumor necrosis factor-alpha, epidermal growth factor, and beta 2-microglobulin levels are elevated in gingival crevicular fluid during human orthodontic tooth movement. J Dent Res. 1996;75(1):562-567. doi:10.1177/00220345960750010801

22. Yamaguchi $M$, Yoshii $M$, Kasai K. Relationship between substance $P$ and interleukin-1beta in gingival crevicular fluid during orthodontic tooth movement in adults. Eur J Orthod. 2006;28(3):241-246. doi:10.1093/ejo/cji100

23. Iwasaki LR, Chandler JR, Marx DB, Pandey JP, Nickel JC. IL-1 gene polymorphisms, secretion in gingival crevicular fluid, and speed of human orthodontic tooth movement. Orthod Craniofac Res. 2009;12(2):129-140. doi:10.1111/j.1601-6343.2009.01446.x

24. Grant M, Wilson J, Rock P, Chapple I. Induction of cytokines, MMP9, TIMPs, RANKL and OPG during orthodontic tooth movement. Eur J Orthod. 2013;35(5):644-651. doi:10.1093/ejo/cjs057

25. AlSwafeeri H, ElKenany W, Mowafy M, Helmy M. Crevicular alkaline phosphatase activity during the application of two patterns of orthodontic forces. J Orthod. 2015;42(1):5-13. doi:10.1179/146531 3314 Y.0000000121 